\title{
Leptin Receptor Deficiency Protects Mice against Chronic Cerebral Hypoperfusion-Induced Neuroinflammation and White Matter Lesions
}

\author{
Yu Du $(\mathbb{D}$, Yufei Song, Xiaojie Zhang, Yan Luo, Wenying Zou, Jiawei Zhang, \\ and Jianliang Fu iD \\ Department of Neurology, Shanghai Jiao Tong University Affiliated Sixth People's Hospital, 600 Yishan Road, \\ Shanghai 200233, China \\ Correspondence should be addressed to Jianliang Fu; fujianliang@163.com
}

Received 30 January 2020; Revised 30 November 2020; Accepted 4 December 2020; Published 18 December 2020

Academic Editor: Michal A. Rahat

Copyright (c) $2020 \mathrm{Yu}$ Du et al. This is an open access article distributed under the Creative Commons Attribution License, which permits unrestricted use, distribution, and reproduction in any medium, provided the original work is properly cited.

\begin{abstract}
Leptin participates in the inflammatory responses in multiple cell types and animal models. Chronic cerebral hypoperfusion (CCH) induces inflammation in the central nervous system (CNS), which acts as one of the main reasons for CCH-induced white matter lesions (WMLs). But whether leptin participates in the pathogenesis of $\mathrm{CCH}$-induced WMLs remains unknown. Therefore, we performed bilateral common carotid artery stenosis (BCAS) to induce $\mathrm{CCH}$ on the leptin receptor- (LepR-) deficient $\mathrm{db} / \mathrm{db}$ mice, aiming to evaluate the possible involvement of leptin in $\mathrm{CCH}$-induced cognitive impairment, WMLs, and neuroinflammation, and further explore the effect of leptin on chronic hypoxia-induced inflammation using the BV2 microglial cell line. After four weeks of BCAS, wild-type mice showed significant working memory deficits, WMLs, activation of microglia and astrocytes, decrease in the number of oligodendrocytes, downregulation of myelin basic protein expression, and increase in the expression of TNF- $\alpha$ and IL-1 $\beta$; however, four weeks of BCAS failed to induce significant changes in the LepR-deficient $\mathrm{db} / \mathrm{db}$ mice but elevated the production of anti-inflammatory cytokines and activated the M2 microglia. We further confirmed that leptin would aggravate the hypoxia-induced proinflammatory cytokine expression in the BV2 microglia cell line. These results suggested that LepR deficiency would protect mice against the $\mathrm{CCH}$-induced cognitive impairment and WMLs by inhibiting glial activation and suppressing proinflammatory responses as well as promoting anti-inflammatory cytokine expression and M2 microglia activation in the white matter.
\end{abstract}

\section{Introduction}

White matter hyperintensities (WMHs) are commonly observed in the brain scanning of the elderly population, which reflect the pathology of white matter lesions (WMLs) [1]. WMLs occur due to chronic cerebral hypoperfusion $(\mathrm{CCH})$ and the subsequent chronic cerebral hypoxia. It could contribute to cognitive decline and even dementia [2]. However, the mechanisms and modulating factors involved in the $\mathrm{CCH}$-induced WHLs are still not fully understood.

$\mathrm{CCH}$ induces chronic inflammation in the central nervous system (CNS), and inflammation might be the mediator of $\mathrm{CCH}$-induced WMLs [3]. Microglia are the immune cells in CNS. They act as the first line of defense against pathogens, clearing up cellular debris, expressing inflammatory cytokines [4]. It was demonstrated that suppressing inflammatory responses and inactivating microglia ameliorated $\mathrm{CCH}$-induced cognitive dysfunction and WMLs [5].

Leptin is predominantly produced by adipose tissue. It functions through the leptin receptor (LepR) and was originally recognized as the modulator of food intake and metabolism. However, growing evidence suggested that leptin also participated in the inflammatory responses and immunity, regulating the production of several cytokines [6]. Leptin also plays an important role in CNS, targeting multiple cell types via LepR [7]. It was confirmed that microglia could express 
LepR and produce proinflammatory cytokines under leptin stimulation [8]. But whether leptin participates in the pathogenesis of $\mathrm{CCH}$-induced WMLs remains unknown.

In this study, we performed bilateral common carotid artery stenosis (BCAS) to induce $\mathrm{CCH}$ in the LepRdeficient $\mathrm{db} / \mathrm{db}$ mice, aiming to evaluate the possible involvement of leptin in $\mathrm{CCH}$-induced cognitive impairment, WMLs, and neuroinflammation, and further explore the effect of leptin on chronic hypoxia-induced inflammation using the BV2 microglial cell line.

\section{Materials and Methods}

2.1. Animals. Male $\mathrm{db} / \mathrm{db}$ mice (BKS.Cg-Dock7m $+/+$ Leprdb/Nju) and wild-type mice (C57BLKS/JNju) were purchased from Nanjing Biomedical Research Institute of Nanjing University (Nanjing, China). Mice were housed in cages with a $12 \mathrm{~h}$ light/dark cycle and were given access to food and water ad libitum. All the surgical and behavioral procedures were conducted according to the National Institutes of Health for the care and use of laboratory animals in scientific investigations and approved by the Ethics of Animal Use in Research Committee of Shanghai Jiaotong University School of medicine. $\mathrm{Db} / \mathrm{db}$ mice have been proved to carry the homozygous spontaneous mutations in the LepR and are resistant to leptin [9]. There were differences in the body weight between $\mathrm{WT}$ and $\mathrm{db} / \mathrm{db}$ mice. The bodyweight of the wild-type mice we used in the experiment was $21.7 \pm$ $3.2 \mathrm{~g}$, and the bodyweight of $\mathrm{db} / \mathrm{db}$ mice was $58.6 \pm 5.1 \mathrm{~g}$.

2.2. Animal Models of Chronic Cerebral Hypoperfusion. Chronic cerebral hypoperfusion $(\mathrm{CCH})$ was induced by bilateral common carotid artery stenosis (BCAS) using microcoils as described elsewhere [10]. These microcoils were made of piano wire with an inner diameter of $0.18 \mathrm{~mm}$, a pitch of $0.50 \mathrm{~mm}$, and a total length of $2.5 \mathrm{~mm}$ (Sawane Spring Co., Shizuoka, Japan). For the BCAS surgery, mice aging 12-16 weeks were anesthetized with $2 \%$ isoflurane and then maintained with $1.5 \%$ isoflurane in $70 \% \mathrm{~N}_{2}$ and $30 \% \mathrm{O}_{2}$, with a heating pad to maintain the rectal temperature within $37 \pm$ $0.5^{\circ} \mathrm{C}$. Through a midline cervical incision, both common carotid arteries (CCAs) were exposed and freed from the vagal nerves. Two 4-0 silk sutures were placed around the distal and proximal parts of each CCA to make a lift. Then, microcoils were rotated around the bilateral CCAs just below the carotid bifurcation. Mice in the sham group simply went through these procedures without applying the microcoils.

2.3. Experimental Groups of Animal Models. Db/db mice and wild-type mice were randomly assigned into four groups as follows: (1) sham-operated wild-type mice (wild-type sham); (2) BCAS-operated wild-type mice (wild-type BCAS); (3) sham-operated $\mathrm{db} / \mathrm{db}$ mice ( $\mathrm{db} / \mathrm{db}$ sham); (4) BCASoperated $\mathrm{db} / \mathrm{db}$ mice $(\mathrm{db} / \mathrm{db}$ BCAS). Mice from the BCASoperated groups were euthanized at three time points for different experiments. After three days and one week of BCAS, mice were directly euthanized for the subsequent analysis. After four weeks of BCAS, mice were executed following the behavioral test.
2.4. Cell Culture and Treatment. The BV2 microglial cell line was purchased from ScienCell (CA, USA) and cultured in MEM medium with $10 \%$ FBS and $1 \%$ penicillin/streptomycin. For normoxia culture, cells were maintained in a humidified incubator containing $95 \%$ air and $5 \% \mathrm{CO}_{2}$ at $37^{\circ} \mathrm{C}$; for hypoxia culture, cells were maintained in a tri-gas incubator (Thermo Fisher Scientific, USA) with oxygen concentration at $1 \%$. BV2 cells were cultured in the normoxia/hypoxia condition with/without the treatment of mouse recombinant leptin (Peprotech, UK) at the concentration of $1 \mu \mathrm{M}$ for $24 \mathrm{~h}$.

2.5. Measurement of the Cerebral Blood Flow (CBF). Under deep anesthesia of isoflurane described above, mice were placed in a ventral recumbent position on a heating pad, and the rectal temperature was maintained between 36.5$37.5^{\circ} \mathrm{C}$. The scalp and the periosteum were removed to expose the skull, and then a thin layer of glycerin was applied on the skull to keep it moisturized. Next, the CBF was monitored through the skull with a laser speckle blood flow imager (Pericam, Sweden) for four min. The region of interest was a $3 \times 5 \mathrm{~mm}^{2}$ rectangle located between the bregma and the lambda, and the measuring height of the laser probe was $10 \mathrm{~cm}$. CBF of mice from different groups was repeatedly recorded at different time points.

2.6. Behavioral Test for Spatial Working Memory. Y-maze test was conducted in the four groups of mice at the end of week four to evaluate spatial working memory. Briefly, each mouse was placed in the center of a Y-shape maze, which consists of three equal arms $(30 \mathrm{~cm}$ long $\times 10 \mathrm{~cm}$ high $\times 8 \mathrm{~cm}$ wide $)$ and was allowed to explore freely within $8 \mathrm{~min}$. Each arm that the mouse entered was recorded, and the exploration of three arms successively was a correct alternation. The percentage of spontaneous alternations was calculated as the number of correct alternations divided by the total number of alternations [the number of correct alternations/(the total number of arms entries-2) $\times 100 \%]$.

2.7. Preparation for Histological Experiments. After four weeks of BCAS or sham operation, wild-type mice and $\mathrm{db} / \mathrm{db}$ mice were anesthetized with pentobarbital sodium and transcardiac perfused with precooled PBS and 4\% PFA (in PBS). The whole brain was harvested and postfixed in $4 \%$ PFA at $4^{\circ} \mathrm{C}$ for $6 \mathrm{~h}$ and subsequently dehydrated in $20 \%$ and $30 \%$ sucrose. After that, the mouse brain was embedded in optimal cutting temperature (OCT) (Sakura, USA) and sliced into consecutive coronal sections (thickness of $10 \mu \mathrm{m}$ or $20 \mu \mathrm{m}$ per section) from bregma $+1.10 \mathrm{~mm}$ to bregma $-0.22 \mathrm{~mm}$ at $-20^{\circ} \mathrm{C}$ in a cryostat (Leica, Germany). These sections were equally divided into three groups according to their location: the anterior, middle, and posterior part of the corpus callosum. Every time one section from each part with the same intervals in each mouse was chosen for the histological experiments.

2.8. Measurement of WMLs. The Klüver-Barrera (KB) staining was used to measure the degree of WMLs. We used $10 \mu \mathrm{m}$ sections with the same intervals from each mouse as mentioned above, and the KB staining was performed as previously described [11]. The severity of white matter lesions 
was determined as normal (grade 0), disarrangement of the nerve fibers (grade 1), formation of marked vacuoles (grade 2 ), or disappearance of myelinated fibers (grade 3 ) in the corpus callosum [12].

2.9. Immunofluorescence Staining. For immunofluorescence staining, $20 \mu \mathrm{m}$ sections were washed with $0.01 \mathrm{M}$ phosphate-buffered saline (PBS) for $3 \times 5 \mathrm{~min}$ and blocked in $5 \%$ normal donkey serum (Jackson, USA) with $0.3 \%$ Triton X-100 (Sigma, USA) for $30 \mathrm{~min}$ at room temperature. The sections were then incubated with goat anti-Glial Fibrillary Acidic Protein (GFAP) antibody (Abcam, UK, 1:500), goat anti-ionized calcium-binding adaptor molecule-1 (Iba1) antibody (Abcam, UK, 1:500), rabbit anti-Olig2 antibody (Proteintech, China, 1:100), rabbit anti-MBP antibody (Abcam, UK, 1:1000), rat anti-CD16/32 (BD Bioscience, USA, $1: 200$ ), or rabbit anti-CD206 (Abcam, UK, $1: 1000$ ) overnight at $4^{\circ} \mathrm{C}$. After washing with PBS for $3 \times 5 \mathrm{~min}$, the sections were then incubated with immunofluorescent secondary antibodies (Abcam, UK, 1:500) for $30 \mathrm{~min}$ in darkness at room temperature. The sections were then washed with PBS for $3 \times 10 \mathrm{~min}$ and incubated with DAPI (CST, USA) for $5 \mathrm{~min}$. Next, the sections were mounted with mounting media (Vector Labs, USA) and imaged with a fluorescence microscope (Olympus, Japan) as soon as possible. Cell numbers, the areas of the corpus callosum, and the mean OD intensities were counted and calculated with ImageJ software.

2.10. Real-Time PCR. Wild-type mice and $\mathrm{db} / \mathrm{db}$ mice with BCAS operation for 3/7/28 days and sham operation were euthanized and transcardiac perfused with precooled PBS solution. The whole brain was then harvested, and the total corpus callosum was dissected under a stereomicroscope on ice. The cerebral cortex was removed as much as possible. The total RNA of the corpus callosum and cultured BV2 cells was extracted using Trizol reagents (Beyotime, China) and reverse-transcripted using the ReverTra Ace qPCR RT Master Mix with gDNA Remover kit according to the manufacturer's protocol (Toyobo, Japan). The real-time PCR was conducted using the SYBR Green Real-time PCR Master Mix kit (Toyobo, Japan) and the ABI 7500 FAST real-time PCR system (Applied Biosystems, USA). The primers are as follows $\left(5^{\prime}-3^{\prime}\right)$ : tumor necrosis factor- $\alpha$ (TNF- $\alpha$ ), forwardCCACCACGCTCTTCTGTCTAC, reverse-AGGGTCTGG GCCATAGAACT; interleukin-10 (IL-10), forward-GCTG CCTGCTCTTACTGACT, reverse-CTGGGAAGTGGGTG CAGTTA; interleukin-1 $\beta$ (IL-1 $\beta$ ), forward-TGCAGCTGG AGAGTGTGGATCCC, reverse-TGTGCTCTGCTTGTGA GGTGCTG; interleukin-6 (IL-6), forward-CTGCAAGAG ACTTCCATCCAG, reverse-AGTGGTATAGACAGGTC TGTTGG; interleukin-4 (IL-4), forward-AACGAGGTCAC AGGAGAAGG, reverse-CTGTGGTGTTCTTCGTTGCT; interleukin-1 receptor antagonist (IL-1Ra), forward-GTGA GACGTTGGAAGGCAGT, reverse-GCATCTTGCAGGGT CTTTTC; GAPDH, forward-GAACGGGAAGCTCACT GG, reverse-GCCTGCTTCACCACCTTCT.GGAGCTGTC ATTAGGGACATCA.

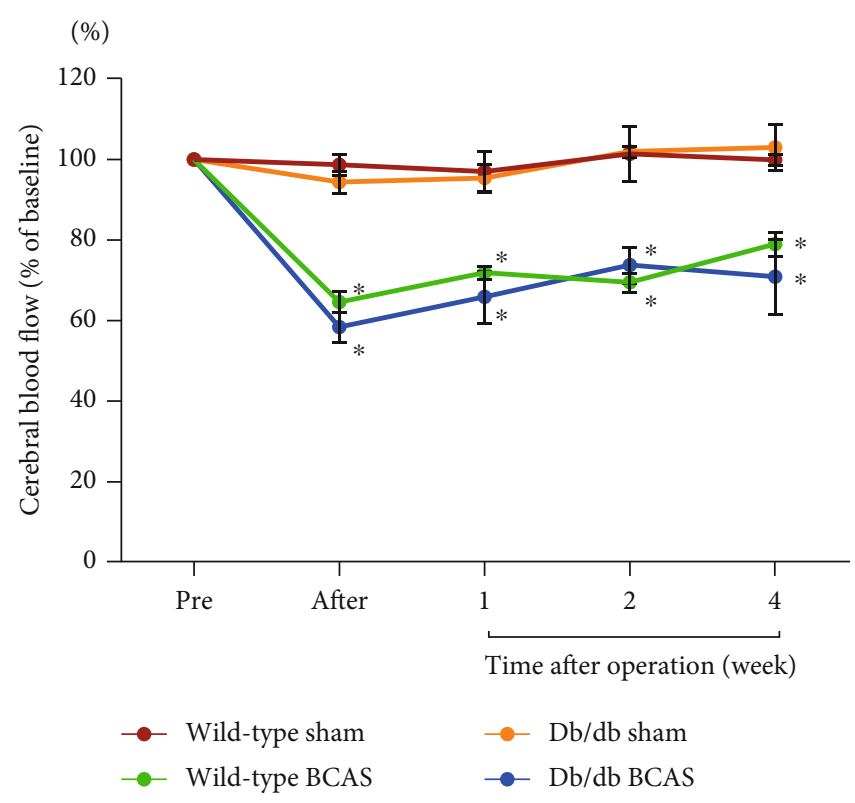

FIgURE 1: Cerebral blood flow (CBF) changes in different groups of mice. $\mathrm{CBF}$ was measured at the following time points: preoperation, right after the operation, one, two, and four weeks after the operation. $\mathrm{CBF}$ value was expressed as the percentage of baseline $\mathrm{CBF}$ value for each animal. Data were presented as the mean \pm SEM. Pre: pre-BCAS/sham operation; after: right after BCAS/sham operation. $N=5,{ }^{*} p<0.05$ compared with sham.

2.11. ELISA. Cell culture supernatants were harvested for ELISA (Multisciences, China) according to the manufacturer's instruction to detect the secreted TNF- $\alpha$ and IL- $1 \beta$ protein levels by BV 2 cells under normoxia and hypoxia conditions with or without the presence of leptin.

2.12. Statistical Analysis. Statistical analysis was performed using IBM SPSS Statistics 22.0, and graphs were plotted using GraphPad Prism 6.0 software. Data were expressed as the mean \pm SEM. The CBF changes were compared using oneway ANOVA with repeated measurements following Fisher's LSD post hoc test; data from the behavioral test, histological examination, immunofluorescent staining, real-time PCR of cell samples, and ELISA were analyzed using one-way ANOVA following Fisher's LSD post hoc test. The statistical analysis methods of inflammatory cytokines real-time PCR data of animal models were explained in the legend.

\section{Results}

3.1. $C B F$ Changes. To verify that $\mathrm{CCH}$ was successfully induced and to examine the magnitude of the blood flow drop-off in mice from different groups, the CBF was measured at five time points: pre-BCAS/sham operation, right after the operation, one week, two weeks, and four weeks after the operation. The CBF values at different time points were expressed as the percentage of the baseline mean perfusion values. It was shown that the CBF values dropped greatly after BCAS in both wild-type and $\mathrm{db} / \mathrm{db}$ groups and recovered overtime gradually but were still lower than 


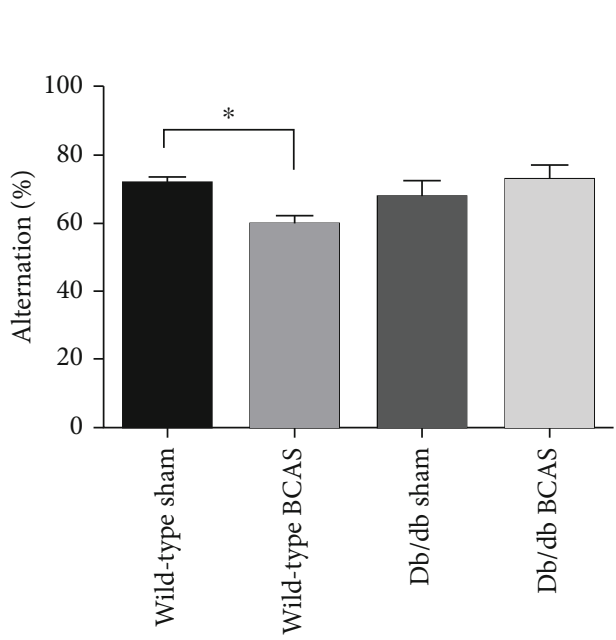

(a)

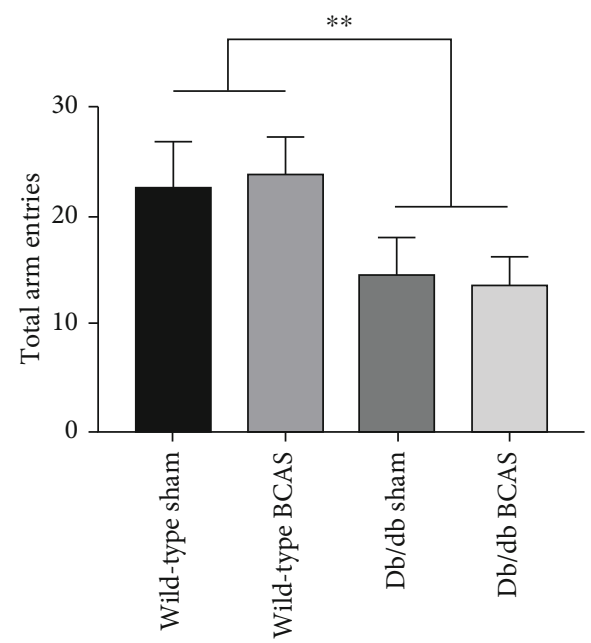

(b)

FIGURE 2: Spatial working memory in mice from different groups. (a) Histogram represented the alternation outcome of mice that underwent the 8-minute Y-maze test. (b) The number of total arm entries of mice from different groups in the Y-maze test. $N=9-14$, data were presented as the mean \pm SEM. ${ }^{*} p<0.05$, and ${ }^{*} p<0.01$.

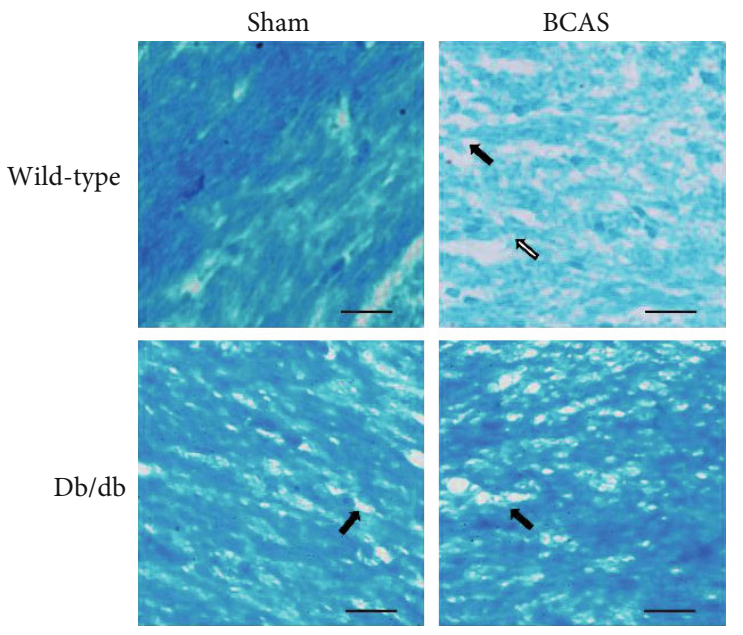

(a)

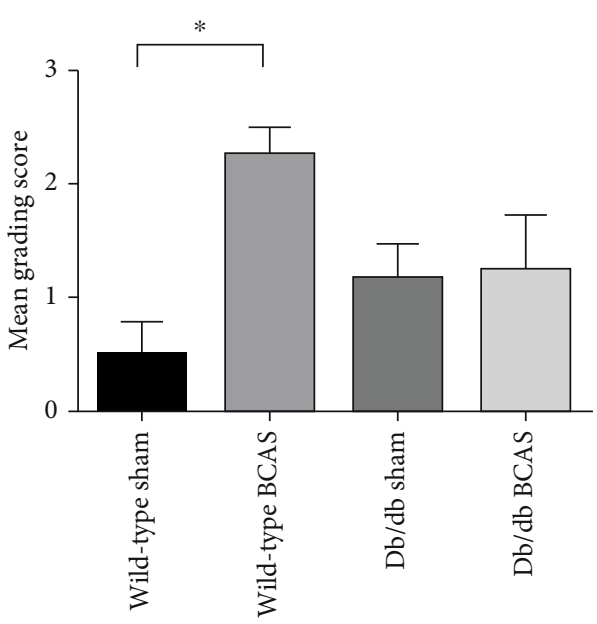

(b)

Figure 3: White matter lesions (WMLs) in wild-type and db/db mice with sham or BCAS operation for four weeks. (a) WMLs were evaluated using Klüver-Barrera $(\mathrm{KB})$ staining. White matter vacuoles were shown by the black arrows, and the disappearance of myelinated fibers was shown by the white arrow. (b) Histogram showing the mean grading scores of WMLs. $N=4-5$, scale bar $=20 \mu \mathrm{m}$, and ${ }^{*} p<0.05$.

preoperation (Figure 1). Compared with sham groups, there was a significant $\mathrm{CBF}$ decline in the BCAS groups both in wild-type and $\mathrm{db} / \mathrm{db}$ mice at any time points after BCAS, while the $\mathrm{CBF}$ values failed to change after the sham operation (Figure 1).

3.2. Spatial Working Memory after BCAS. Given the same scale of CBF decline among wild-type mice and $\mathrm{db} / \mathrm{db}$ mice, it was then explored whether the CBF decline would lead to a certain cognitive deficit. After four weeks of BCAS, we performed a Y-maze test to evaluate the spatial working memory in mice among different groups. Impaired spatial working memory was observed in wild-type mice after four weeks of BCAS, as demonstrated by a reduction in the percentage of alternation behavior. Interestingly, compared with shamoperated $\mathrm{db} / \mathrm{db}$ mice, there were no further cognitive deficits in $\mathrm{db} / \mathrm{db}$ mice with BCAS (Figure 2(a)). The number of total arm entries within $8 \mathrm{~min}$ of $\mathrm{db} / \mathrm{db}$ mice was lower than wildtype mice, and there was no difference between BCAS and sham operation (Figure 2(b)). These results suggested that $\mathrm{db} / \mathrm{db}$ mice that carried the dysfunctional LepR mutation and were resistant to leptin might be protected from the $\mathrm{CCH}$ induced cognitive impairment.

3.3. Histological Changes in White Matter. WMLs in wildtype and $\mathrm{db} / \mathrm{db}$ mice with the sham operation or four-week BCAS were determined by KB staining. Four weeks of BCAS induced tremendous lesions in the corpus callosum of wild- 


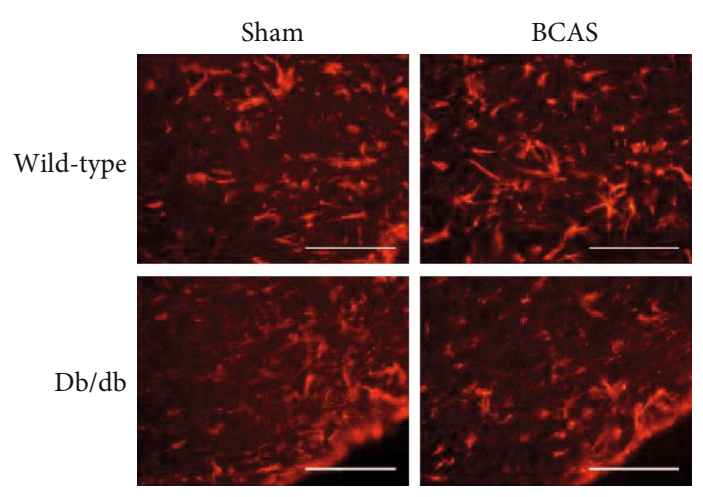

(a)

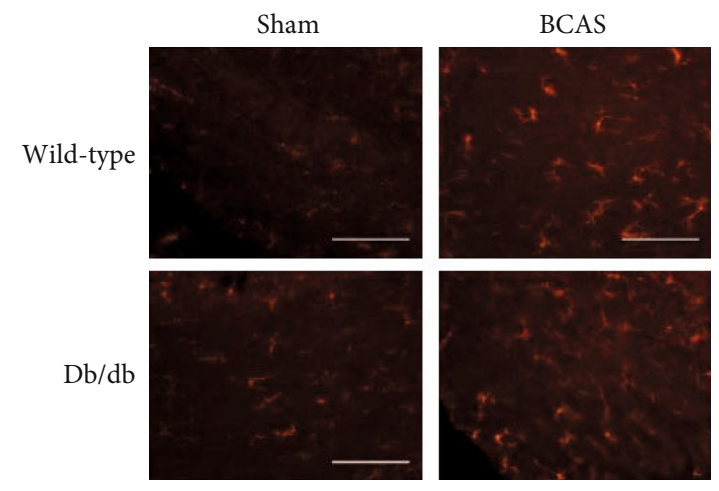

(c)

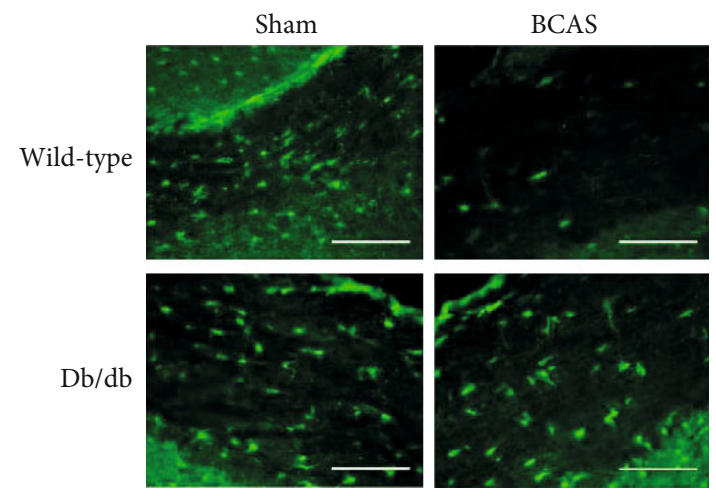

(e)

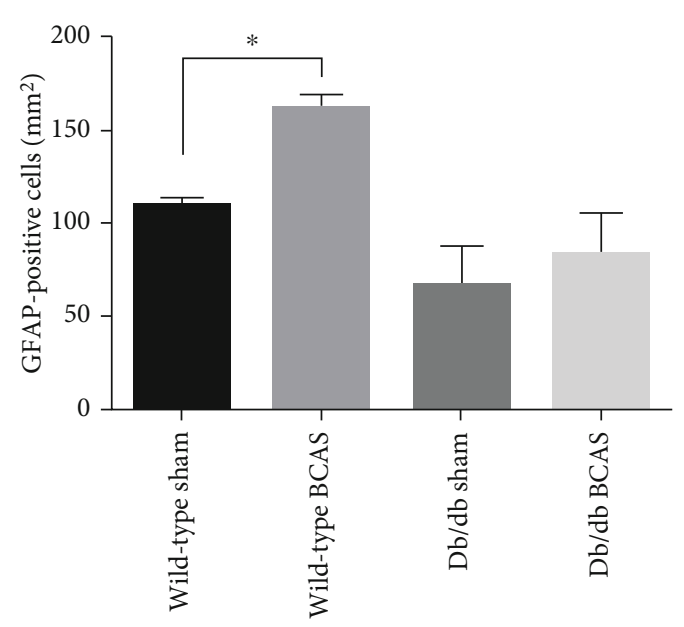

(b)

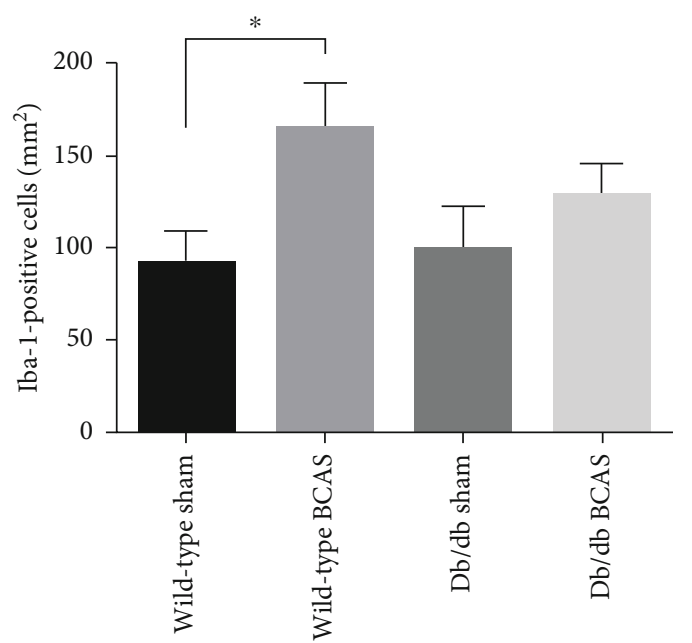

(d)

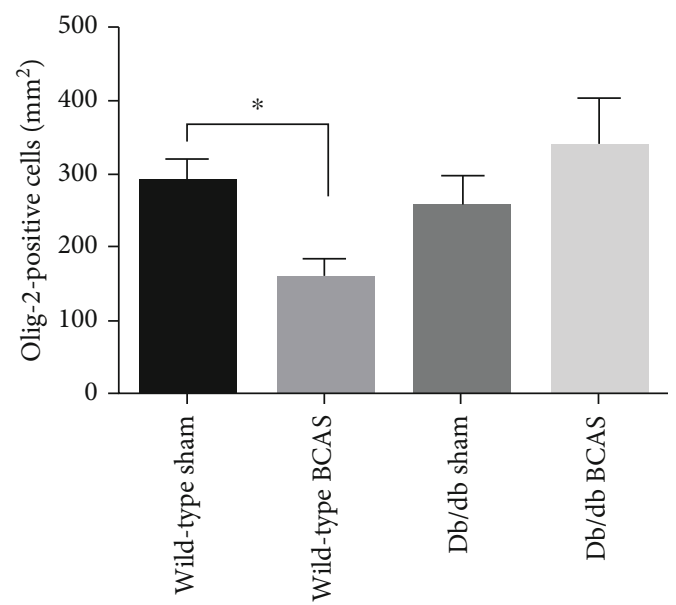

(f)

FIGURE 4: Immunofluorescence staining of glial cells in the corpus callosum of wild-type and db/db mice with sham or BCAS operation for four weeks. (a) Representative images of GFAP-positive astrocytes. (b) Histogram of GFAP-positive astrocytes density in different groups. (c) Representative images of Iba-1-positive microglia. (d) Histogram of Iba-1-positive microglia density in different groups. (e) Representative images of Olig-2-positive oligodendrocytes. (f) Histogram of Olig-2-positive oligodendrocytes density in different groups. $N=3-5$, scale bar $=100 \mu \mathrm{m}$, and ${ }^{*} p<0.05$. 


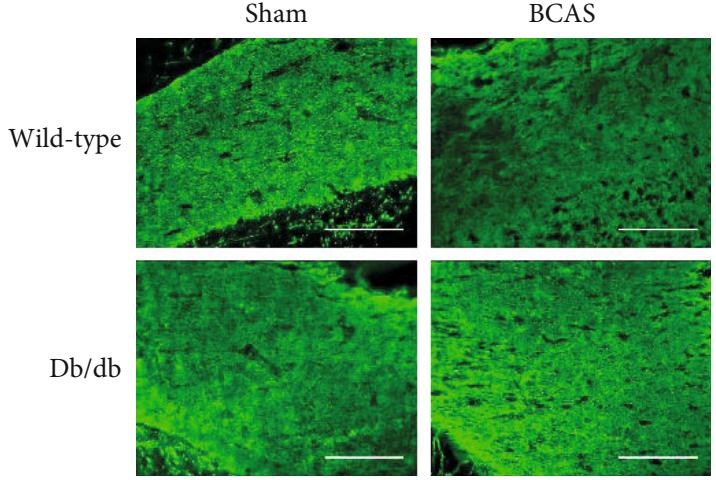

(a)

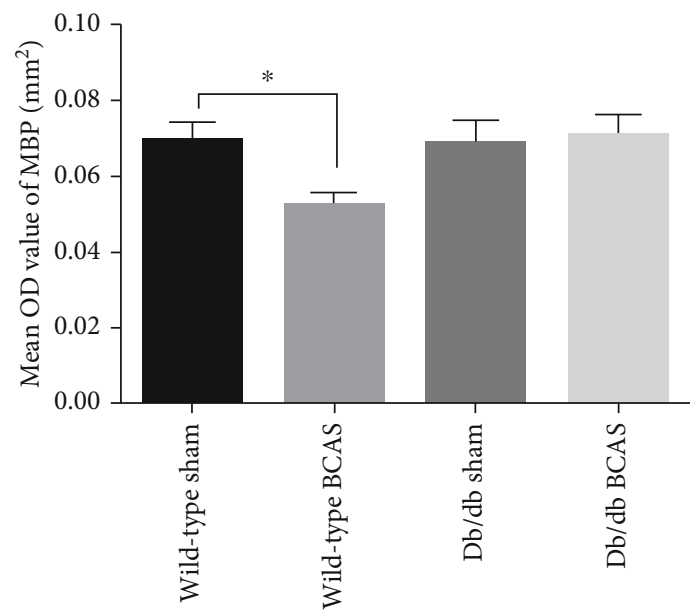

(b)

Figure 5: MBP expression in the corpus callosum of wild-type and db/db mice with sham or BCAS operation for four weeks. (a). Immunofluorescence staining of MBP. (b). Histogram showed the mean OD value of MBP in mice from different groups. $N=3-5$, scale bar $=100 \mu \mathrm{m}$, and ${ }^{*} p<0.05$.

type mice, such as disarrangement of nerve fibers, formation of white matter vacuoles, and even the disappearance of myelinated fibers (Figure 3). There was a slight formation of white matter vacuoles in sham-operated $\mathrm{db} / \mathrm{db}$ mice. However, comparing with sham-operated $\mathrm{db} / \mathrm{db}$ mice, $\mathrm{db} / \mathrm{db}$ mice that underwent four weeks of BCAS did not show any further WMLs (Figure 3).

3.4. Changes of Glial Cells in White Matter. Since there were not significant WMLs in $\mathrm{db} / \mathrm{db}$ mice after BCAS as in wildtype mice, we further explored the glial activation in mice from different groups. The number of Iba-1-positive microglia (Figures 4(a) and 4(b)) significantly increased in wild-type mice after BCAS. In contrast, the amount of microglia in sham-operated $\mathrm{db} / \mathrm{db}$ mice and $\mathrm{db} / \mathrm{db}$ mice after BCAS revealed no difference. The same trend was observed in the number of GFAP-positive astrocytes (Figures 4(c) and $4(d))$. As is known, oligodendrocytes form the sheath of myelin fibers and play an important role in the normal functioning of white matter. The changes of Olig-2-positive oligodendrocytes were then examined (Figures 4(e) and 4(f)) by immunofluorescence staining. Oligodendrocytes decreased dramatically after BCAS in wild-type mice, associated with poor cognitive performance in the Y-maze test. But there was no evident shrinkage of the oligodendrocyte population in $\mathrm{db} / \mathrm{db}$ mice after BCAS. These results corresponded to the behavior and histological tests, indicating that LepR deficiency might protect mice from the $\mathrm{CCH}$-induced significant WMLs and cognitive deficits as occurred in wild-type mice through inactivating glial cells in the white matter.

3.5. Changes in MBP Expression. To further verify the different responses of wild-type mice and $\mathrm{db} / \mathrm{db}$ mice to BCAS in terms of demyelination, the expression of MBP was examined by immunofluorescence staining. Consistent with the changes in oligodendrocytes, it was shown that the MBP level severely decreased in wild-type mice after four weeks of
BCAS. However, there was still no significant change in BCAS-operated $\mathrm{db} / \mathrm{db}$ mice than sham (Figure 5).

3.6. Changes in the Expression of Proinflammatory and AntiInflammatory Cytokines and M1/M2 Phenotype in Corpus Callosum. A previous study indicated that leptin would facilitate proinflammatory cytokine production in microglia [8]. We speculated that lacking LepR might modulate the cytokine expression profile in $\mathrm{db} / \mathrm{db}$ mice after BCAS and regulate the cognitive behavior and histological performance. The corpus callosum of wild-type mice and $\mathrm{db} / \mathrm{db}$ mice with the sham operation and BCAS operation for $3 / 7 / 28$ days was dissected, and the expression of several kinds of representative proinflammatory cytokines (TNF- $\alpha$, IL- $1 \beta$, and IL-6) and anti-inflammatory cytokines (IL-10, IL-4, and IL-1Ra) was examined using real-time PCR. The expression of TNF- $\alpha$ in wild-type mice increased significantly after three days of BCAS, then it decreased to the same level as the sham group, while TNF- $\alpha$ expression level did not elevate after BCAS in $\mathrm{db} / \mathrm{db}$ mice at any time point (Figure $6(\mathrm{a})$ ). IL-1 $\beta$ was elevated at all time points after BCAS in wild-type mice, but it did not increase in $\mathrm{db} / \mathrm{db}$ mice after BCAS as much as the wild-type, with the only increase after seven days of BCAS (Figure 6(b)). The expression levels of IL-6 in different groups were unaltered (Figure 6(c)). As for the antiinflammatory cytokines (Figures $6(\mathrm{~d})-6(\mathrm{f})$ ), the expression levels of IL-10, IL-4, and IL-1Ra did not change in the wildtype mice after BCAS at any time points, whereas in the $\mathrm{db} / \mathrm{db}$ mice, all of them exhibited upregulation at certain time points. These results indicated that LepR deficiency might protect mice from $\mathrm{CCH}$-induced brain damage through suppressing proinflammatory cytokine expression and promoting anti-inflammatory cytokine expression. Then, the microglial phenotypes were analyzed by immunofluorescence staining. It was found that the amount of both CD16/32-positive M1 microglia and CD206-positive M2 microglia in the corpus callosum was increased in 


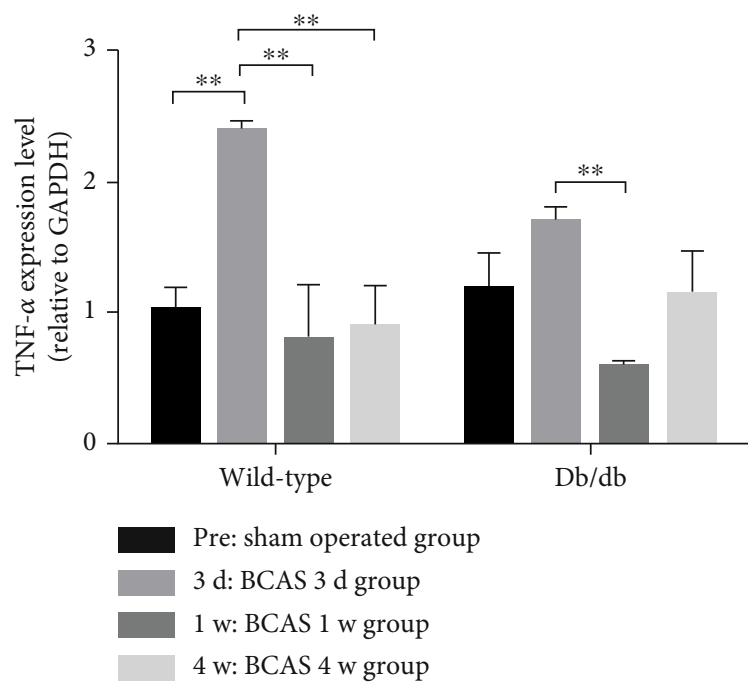

(a)

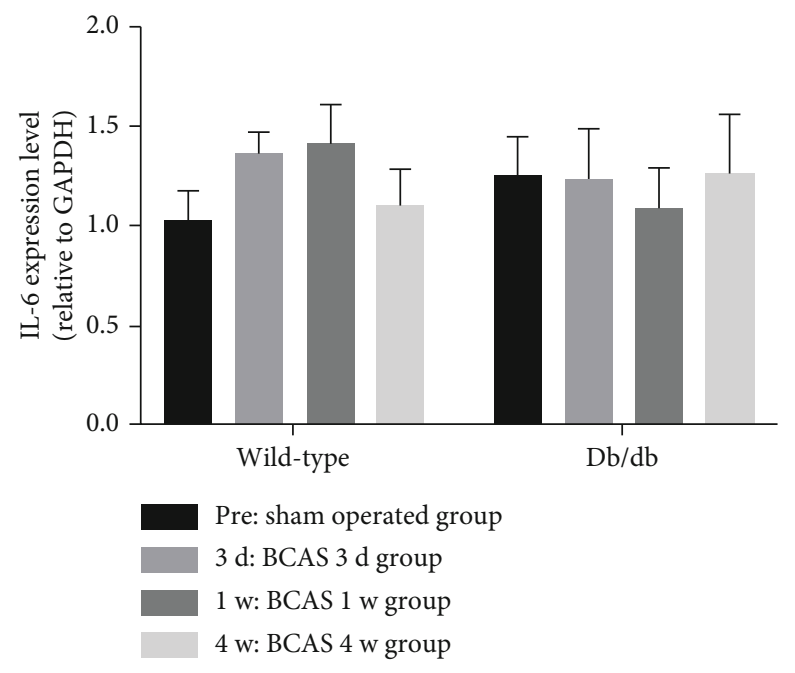

(c)

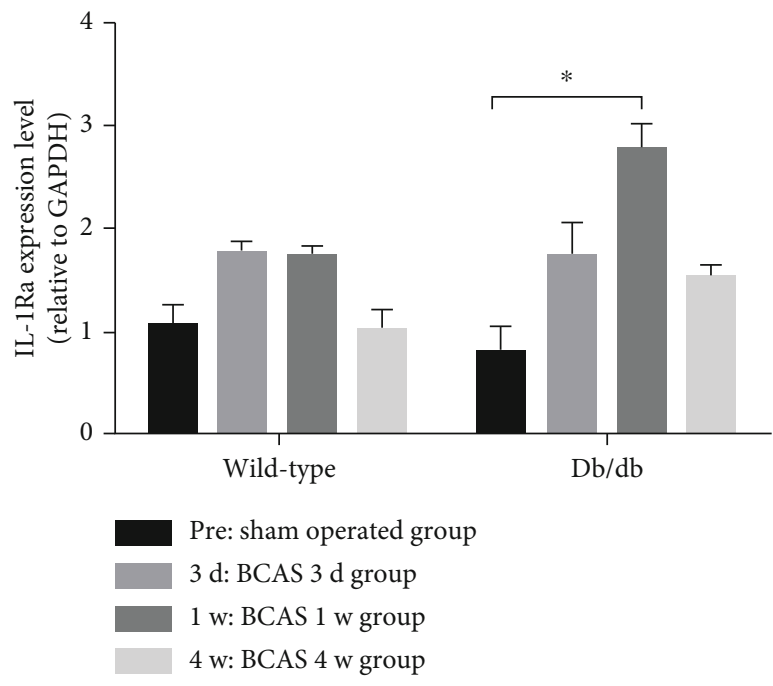

(e)

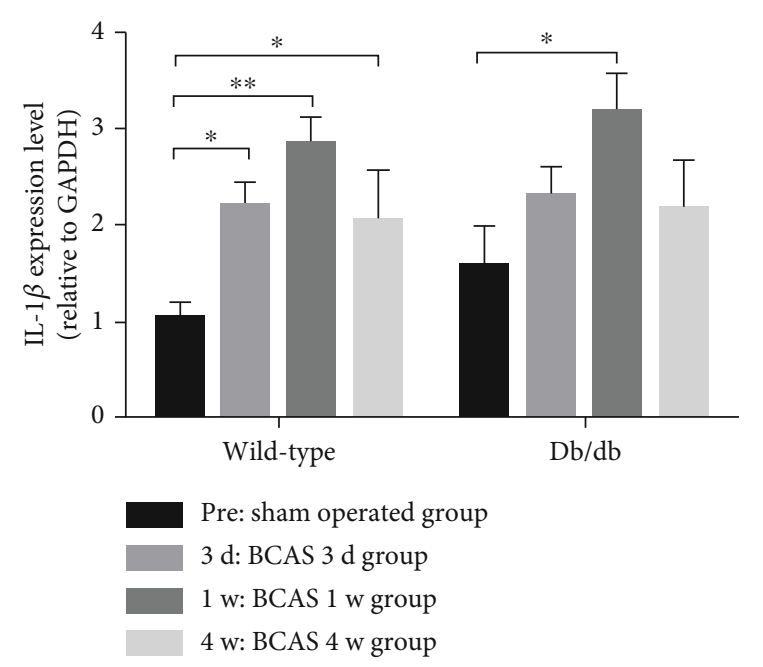

(b)

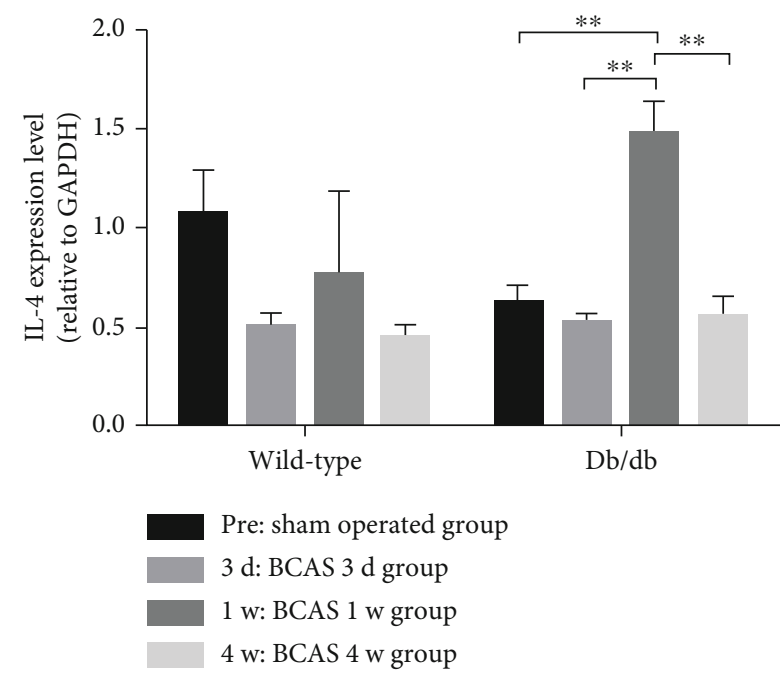

(d)

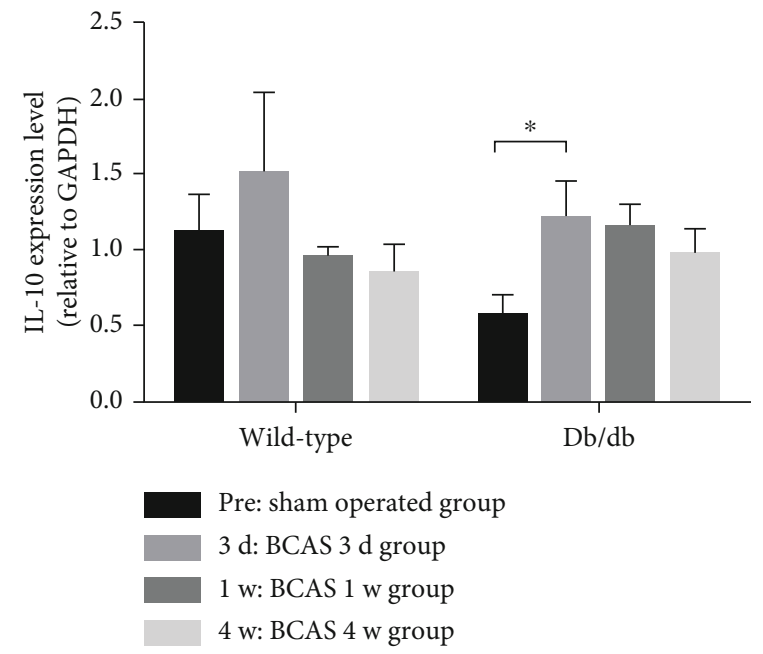

(f)

Figure 6: Continued. 


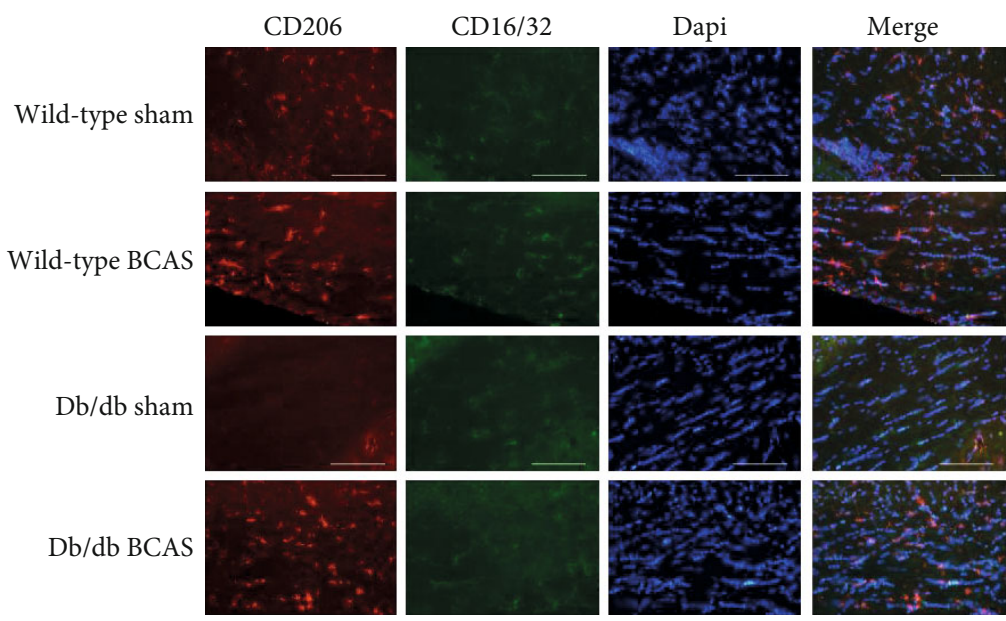

(g)

FIGURE 6: Expression of inflammatory cytokines in the corpus callosum of wild-type and db/db mice with sham or BCAS operation for four weeks. Relative mRNA expression levels of proinflammatory cytokines TNF- $\alpha$ (a), IL-1 $\beta$ (b), and IL-6 (c), and anti-inflammatory cytokines IL-4 (d), IL1Ra (e), and IL-10 (f) against GAPDH were examined by real-time PCR. M1/M2 microglial phenotypes in the mice corpus callosum were examined by immunofluorescence staining. Anti-CD16/32 antibodies were used to label M1-positive microglia, and antiCD206 antibodies were used to label M2-positive microglia (g). In (a-d), comparisons between different time points were analyzed using Fisher's LSD test; in (e), comparison between different time points was analyzed using Dunnett's test; in (f), comparison between different time points in the wild-type group was analyzed using Kruskal Wallis test, and comparison between different time points in $\mathrm{db} / \mathrm{db}$ group was analyzed using Fisher's LSD test. $N=3-5$, scale bar $=100 \mu \mathrm{m},{ }^{*} p<0.05$, and ${ }^{*}{ }^{*} p<0.01$. Wild-type: wild-type mice, $\mathrm{Db} / \mathrm{db}$ : $\mathrm{db} / \mathrm{db}$ mice.

wild-type mice after BCAS operation; however, in $\mathrm{db} / \mathrm{db}$ mice, the amount of CD206-positive M2 microglia significantly increased after BCAS operation, but CD16/32-positive M1 microglia were inhibited (Figure 6(g)).

\subsection{Leptin Promoted Proinflammatory Cytokine Expression} in BV2 Cells under Hypoxia Condition. As we have shown that deficiency of LepR suppressed the $\mathrm{CCH}$ induced proinflammatory cytokines expression, we then wanted to confirm whether leptin would promote the proinflammatory cytokine expression in microglia under hypoxia condition in return. We cultured BV2 cells under normoxia/hypoxia (1\% oxygen concentration) conditions with/without leptin $(1 \mu \mathrm{M})$ treatment for $24 \mathrm{~h}$ and harvested cells for real-time PCR and ELISA examination. We found that hypoxia stimulated TNF- $\alpha$ and IL- $1 \beta$ mRNA and protein expression in BV2 cells, and leptin treatment further promoted the expression of TNF- $\alpha$ and IL- $1 \beta$; however, hypoxia failed to modify the expression of IL- 6 in BV2 cells, although leptin treatment triggered the expression of IL-6 (Figure 7).

\section{Discussion}

Previous studies indicated that leptin would facilitate proinflammatory cytokine production in microglia and other cell types as well as animal models $[8,13]$. Microglia are the major source of cytokine production in the CNS, and $\mathrm{CCH}$ induced neuroinflammation is believed to be an important pathogenic mechanism of $\mathrm{CCH}$-induced WMLs and cognitive impairment. It brought us to think about the potential role of leptin in the $\mathrm{CCH}$-induced WMLs. In this study, we first provide evidence that leptin signaling plays a pivotal role in the pathogenesis of CCH-induced WMLs. We found that
LepR deficiency would protect mice from the CCH-induced cognitive impairment and WMLs by inhibiting glial activation and suppressing proinflammatory cytokine expression as well as promoting anti-inflammatory cytokine expression in the white matter. And we further confirmed that leptin would aggravate the hypoxia-induced proinflammatory cytokine expression in the BV2 microglia cell line.

We successfully induced mild $\mathrm{CCH}$ by performing BCAS for four weeks in wild-type mice and $\mathrm{db} / \mathrm{db}$ mice, revealed by the $\mathrm{CBF}$ reduction. We chose the $\mathrm{Y}$-maze test and the 8minute alternation protocol to test the spatial working memory of mice from different groups. BCAS would lead to poor $\mathrm{Y}$-maze 8 -minute alternation performance $[14,15]$, which was further verified in our study. Although many studies have addressed the neuronal and cognitive-behavioral features of $\mathrm{db} / \mathrm{db}$ mice, Sharma et al. suggested that there was no spatial working memory deficit in $\mathrm{db} / \mathrm{db}$ mice [16]. In our study, we confirmed that $\mathrm{db} / \mathrm{db}$ mice had no spatial working memory deficit compared with wild-type mice and found that $\mathrm{db} / \mathrm{db}$ mice after four weeks of BCAS showed no further spatial working memory impairment. $\mathrm{Db} / \mathrm{db}$ mice presented several characteristics of metabolic syndrome, such as overweight and high blood glucose. But we did not observe connections between high blood glucose and chronic cerebral hypoperfusion-induced spatial working memory impairment. So, we did not emphasize the overweight of $\mathrm{db} / \mathrm{db}$ mice. Concerning that BCAS cerebral hypoperfusion model mainly affects the integrity and function of the corpus callosum and leads to spatial working memory dysfunction [17], we chose Y-maze 8-minute alternation to evaluate the cognitive performance in different groups as previously reported [18]. The results indicated that mild $\mathrm{CCH}$ decline could not induce a cognitive decline in the LepR-deficient 


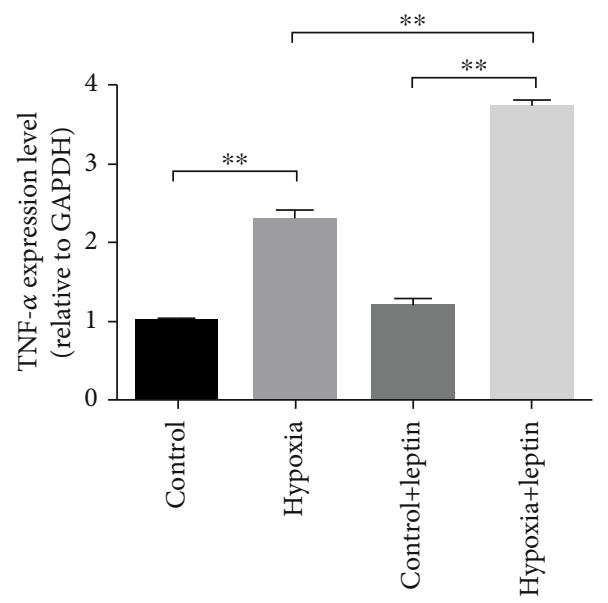

(a)

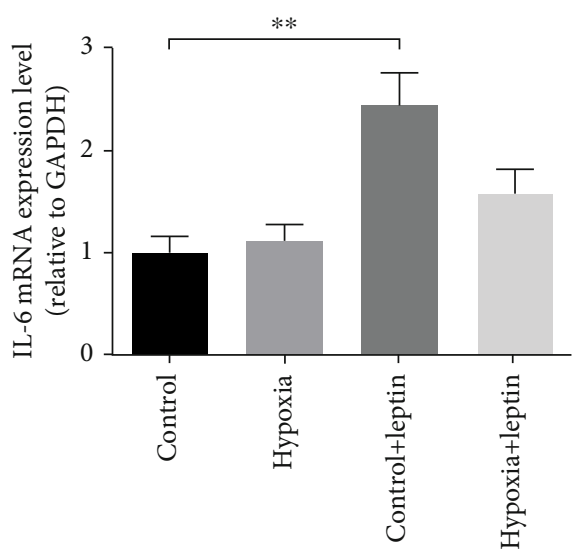

(c)

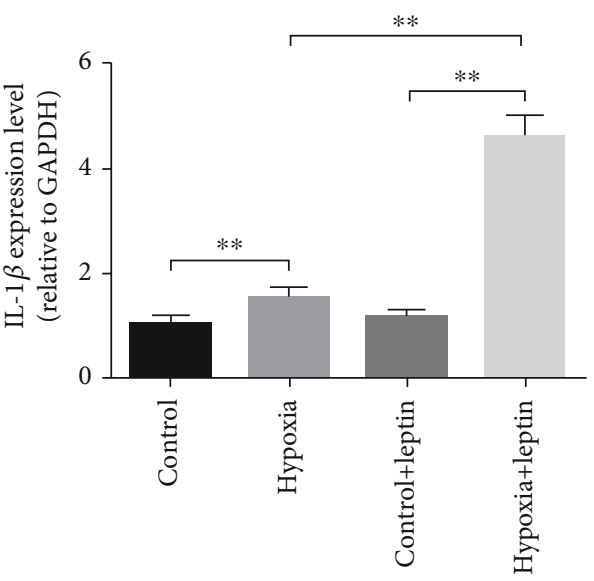

(b)

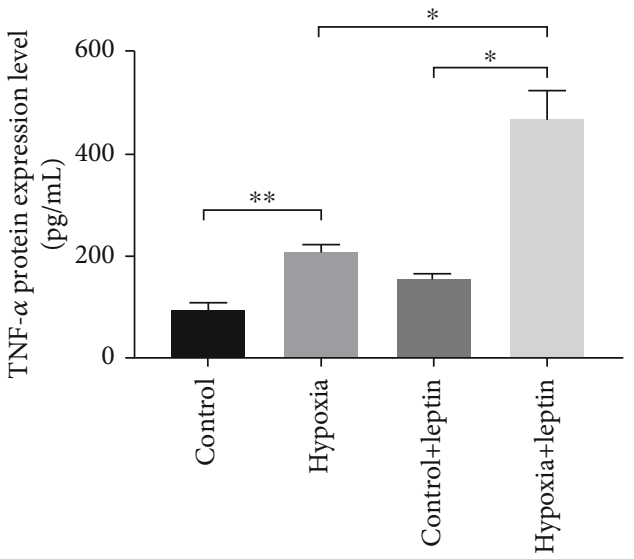

(d)

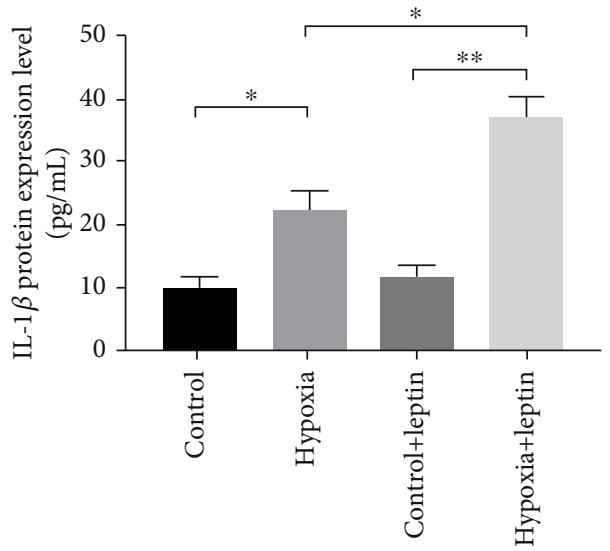

(e)

FIGURE 7: Expression of proinflammatory cytokines TNF- $\alpha$ (a) and IL-1 $\beta$ (b) in BV2 cells under normoxia/hypoxia conditions with/without leptin treatment for 24 hours. The mRNA expression levels of TNF- $\alpha$ (a), IL-1 $\beta$ (B), and IL-6 (c) were examined by real-time PCR, and the protein expression levels of TNF- $\alpha$ (d) and IL- $1 \beta$ (e) were examined by ELISA. $N=3, p<0.05$, and ${ }^{* *} p<0.01$.

$\mathrm{db} / \mathrm{db}$ mice as it did in the wild-type mice, and LepR deficiency might protect the $\mathrm{db} / \mathrm{db}$ mice from the severe damage that $\mathrm{CCH}$ would have caused. We noted that the number of total arm entries of $\mathrm{db} / \mathrm{db}$ mice was lower than wild-type mice. We speculated that this was because of the lower motor ability of $\mathrm{db} / \mathrm{db}$ mice due to overweight.
The activation of microglia and astrocytes and the decline of oligodendrocyte density and MBP expression are the common features of CCH-induced WMLs [19]. Activated microglia would participate in the white matter damage through releasing proinflammatory cytokines such as TNF$\alpha$ and IL-1 $\beta$ [5]. However, the lack of LepR might lead to 
poor microglia and astrocyte responsiveness against disturbances such as trauma, hypoxia, and inflammation [20, 21]. In our study, we found that LepR dysfunction protected the mice from the $\mathrm{CCH}$-induced histological damages in the white matter, ameliorating the $\mathrm{CCH}$-stimulated expression of proinflammatory cytokines such as TNF- $\alpha$ and IL- $1 \beta$, and further upregulated the expression of antiinflammatory cytokines such as IL-4, IL-10, and IL-1Ra. As microglia can be simplistically divided into the proinflammatory M1 microglia and the anti-inflammatory M2 microglia [22], the microglial phenotypes were then examined using the phenotype markers: CD16/32 (M1) and CD206 (M2). Consistent with the cytokine expression profile, the antiinflammatory M2 microglia in $\mathrm{db} / \mathrm{db}$ mice dominated after the BCAS operation. Furthermore, cell experiments revealed that leptin would further upregulate the hypoxia-induced proinflammatory cytokine expression. These results manifested that lack of LepR, which would mediate the proinflammatory signaling pathway in the CNS, prevented the proinflammatory microglia's participation in the pathogenesis of white matter damage. Nevertheless, the mechanisms through which LepR stimulation and hypoxia work synergistically to induce TNF- $\alpha$ and IL- $1 \beta$ production in BV2 cells remain to be further elucidated. It was reported that in Raw 264.7 cells, phospholipase D1-JNK pathway would mediate the leptin-induced TNF- $\alpha$ expression [23].

There are certain limitations in our study that we will address in our future experiments. We failed to provide evidence of whether the leptin receptor knockdown in the BV2 cells would mimic the specific changes of cytokine production observed in this study in vivo. Moreover, the outcomes of this study are all observational, and the molecular mechanisms of leptin modulating cytokine production remain to be elucidated.

\section{Data Availability}

The data used to support the findings of this study are available from the corresponding author upon request.

\section{Disclosure}

Its publication is approved by all authors.

\section{Conflicts of Interest}

The authors declare no competing interests.

\section{Authors' Contributions}

Yu Du and Yufei Song contributed equally to this work.

\section{Acknowledgments}

This study was supported by the Project of National Natural Science Foundation of China (No. 81871103, No. 81672243, and No.81870952).

\section{References}

[1] M. S. Fernando, J. E. Simpson, F. Matthews et al., "White matter lesions in an unselected cohort of the elderly," Stroke, vol. 37, no. 6, pp. 1391-1398, 2006.

[2] N. D. Prins and P. Scheltens, "White matter hyperintensities, cognitive impairment and dementia: an update," Nature Reviews Neurology, vol. 11, no. 3, pp. 157-165, 2015.

[3] J. Duncombe, A. Kitamura, Y. Hase, M. Ihara, R. N. Kalaria, and K. Horsburgh, "Chronic cerebral hypoperfusion: a key mechanism leading to vascular cognitive impairment and dementia. Closing the translational gap between rodent models and human vascular cognitive impairment and dementia," Clinical Science, vol. 131, no. 19, pp. 2451-2468, 2017.

[4] V. H. Perry, J. A. R. Nicoll, and C. Holmes, "Microglia in neurodegenerative disease," Nature Reviews Neurology, vol. 6, no. 4, pp. 193-201, 2010.

[5] J. Miyanohara, M. Kakae, K. Nagayasu et al., "TRPM2 channel aggravates CNS inflammation and cognitive impairment via activation of microglia in chronic cerebral hypoperfusion," The Journal of Neuroscience, vol. 38, no. 14, pp. 3520-3533, 2018.

[6] G. Fantuzzi and R. Faggioni, "Leptin in the regulation of immunity, inflammation, and hematopoiesis," Journal of Leukocyte Biology, vol. 68, no. 4, pp. 437-446, 2000.

[7] M. M. Scott, J. L. Lachey, S. M. Sternson et al., "Leptin targets in the mouse brain," Journal of Comparative Neurology, vol. 514, no. 5, pp. 518-532, 2009.

[8] C.-H. Tang, D. Y. Lu, R. S. Yang et al., "Leptin-induced IL-6 production is mediated by leptin receptor, insulin receptor substrate-1, phosphatidylinositol 3-kinase, Akt, NF- $\kappa \mathrm{B}$, and p300 pathway in microglia," The Journal of Immunology, vol. 179, no. 2, pp. 1292-1302, 2007.

[9] K. P. Hummel, M. M. Dickie, and D. L. Coleman, "Diabetes, a new mutafton in the mouse," Science, vol. 153, no. 3740, pp. 1127-1128, 1966.

[10] M. Shibata, R. Ohtani, M. Ihara, and H. Tomimoto, "White matter lesions and glial activation in a novel mouse model of chronic cerebral hypoperfusion," Stroke, vol. 35, no. 11, pp. 2598-2603, 2004.

[11] H. Klüver and E. Barrera, "A method for the combined staining of cells and fibers in the nervous system*," Journal of Neuropathology \& Experimental Neurology, vol. 12, no. 4, pp. 400-403, 1953.

[12] W. Duan, L. Gui, Z. Zhou et al., "Adenosine $A_{2 A}$ receptor deficiency exacerbates white matter lesions and cognitive deficits induced by chronic cerebral hypoperfusion in mice," Journal of the Neurological Sciences, vol. 285, no. 1-2, pp. 39-45, 2009.

[13] W.-H. Yang, S. C. Liu, C. H. Tsai et al., "Leptin induces IL-6 expression through OBRl receptor signaling pathway in human synovial fibroblasts," PLoS One, vol. 8, no. 9, article e75551, 2013.

[14] K. Washida, M. Ihara, K. Nishio et al., "Nonhypotensive dose of telmisartan attenuates cognitive impairment partially due to peroxisome proliferator-activated receptor- $\gamma$ activation in mice with chronic cerebral hypoperfusion," Stroke, vol. 41, no. 8, pp. 1798-1806, 2010.

[15] T. Maki, A. Morancho, P. M.-S. Segundo et al., "Endothelial progenitor cell secretome and oligovascular repair in a mouse model of prolonged cerebral hypoperfusion," Stroke, vol. 49, no. 4, pp. 1003-1010, 2018. 
[16] A. N. Sharma, K. M. Elased, T. L. Garrett, and J. B. Lucot, "Neurobehavioral deficits in $\mathrm{db} / \mathrm{db}$ diabetic mice," Physiology \& Behavior, vol. 101, no. 3, pp. 381-388, 2010.

[17] C. Sarti, L. Pantoni, L. Bartolini, and D. Inzitari, "Cognitive impairment and chronic cerebral hypoperfusion: what can be learned from experimental models," Journal of the Neurological Sciences, vol. 203-204, pp. 263-266, 2002.

[18] K. Washida, M. Ihara, K. Nishio et al., "Nonhypotensive dose of telmisartan attenuates cognitive impairment partially due to peroxisome proliferator-activated receptor-gamma activation in mice with chronic cerebral hypoperfusion," Stroke, vol. 41, no. 8, pp. 1798-1806, 2010.

[19] Y.-F. Dong, K. Kataoka, K. Toyama et al., "Attenuation of brain damage and cognitive impairment by direct renin inhibition in mice with chronic cerebral hypoperfusion," Hypertension, vol. 58, no. 4, pp. 635-642, 2011.

[20] R. Kumari, L. B. Willing, J. K. Krady, S. J. Vannucci, and I. A. Simpson, "Impaired wound healing after cerebral hypoxia-ischemia in the diabetic mouse," Journal of Cerebral Blood Flow \& Metabolism, vol. 27, no. 4, pp. 710-718, 2006.

[21] R. Kumari, L. B. Willing, S. D. Patel et al., “The PPAR- $\gamma$ agonist, darglitazone, restores acute inflammatory responses to cerebral hypoxia-ischemia in the Diabeticob/obMouse," Journal of Cerebral Blood Flow \& Metabolism, vol. 30, no. 2, pp. 352-360, 2009.

[22] Y. Tang and W. Le, "Differential roles of M1 and M2 microglia in neurodegenerative diseases," Molecular Neurobiology, vol. 53, no. 2, pp. 1181-1194, 2016.

[23] S. M. Lee, H. J. Choi, C. H. Oh, J. W. Oh, and J. S. Han, "Leptin increases TNF- $\alpha$ expression and production through phospholipase D1 in Raw 264.7 cells," PLoS One, vol. 9, no. 7, article e102373, 2014. 